\title{
LOWEST EXCITED-STATE IMPURITY BINDING ENERGY IN InGaN/GaN PARABOLIC QWW: MAGNETIC FIELD EFFECT
}

\author{
HADDOU EL GHAZI \\ LPS, Faculty of sciences, Dhar EL Mehrez, \\ B.P 1796 Atlas Fez, Morocco \\ and \\ Special Mathematics, CPGE Kenitra, \\ Chakib Arsalane Street, Kenitra Morocco \\ ANOUAR JORIO AND IZEDDINE ZORKANI \\ LPS, Faculty of sciences, Dhar EL Mehrez, \\ B.P 1796 Atlas Fez, Morocco
}

Email: hadghazi@gmail.com

Received 08 August 2013;; revised manuscript received 26 August 2013 Accepted for publication 28 August 2013

\begin{abstract}
In this paper, we have investigated the magnetic field effect on the lowest excitedstate binding energy of hydrogenic shallow-donor impurity in wurtzite (In, Ga)N/GaN parabolic transversal-section quantum-well wire $(P Q W W)$ using the finite-difference method within the quasione-dimensional effective potential model. The calculations are performed within the framework of the effective mass approximation. A cylindrical $Q W W$ effective radius is taken into account to describe the lateral confinement strength. The numerical results show that: (i) the probability density is the largest on a circularity whose radius is the effective radius and (ii) the lowest excited-state binding energy is the largest when an impurity is located on this circularity while it starts to decrease as the impurity is away from the circularity.
\end{abstract}

\section{INTRODUCTION}

In the last few decades, there have been several studies concerning hydrogenic impurities in low-dimensional semiconductors such as quantum well (QW), quantum well wire (QWW) and quantum dot (QD) under external fields which are an interesting probe to study the physical properties both from theoretical and technological points of view [1-19]. Using the potential morphing method within the effective mass approximation, Baskoutas et al. [20-23] have investigated the effect of external electric and magnetic fields on the binding energy in GaAs-GaAlAs inverse parabolic quantum well and quantum dot. Niculescu and coworkers [27] have examined the magnetic field effect on the binding energy inGaAs / $\mathrm{Al}_{0.3} \mathrm{Ga}_{0.7}$ As cylindrical QWW. It has been found that the binding energy increases as a function of the applied magnetic field and the influence of the latter is more pronounced for bigger wire radii and for on-center impurity. The same behavior has been reported by Zounoubi and cowerkers [24] which they have calculated the binding 
energy and the polarizability of shallow-donor confined in GaAs QWW with a rectangular cross-section in the presence of an axial magnetic field. Aktas and coworkers $[13,14]$ have estimated the binding energy of shallow donor impurity under both electric and magnetic fields in a coaxial GaAs $/ \mathrm{Ga}_{x} \mathrm{Al}_{1-x} \mathrm{As}$ QWW. They have calculated the binding energy as a function of the impurity position and barrier thickness for various values of electric and magnetic fields. An et al. [26] have investigated the properties of hydrogenic impurities in a parabolic GaAs QWW in the presence of magnetic field. In the same sense, Kasapoglu et al. [28] have calculated the binding energy in the single square quantum well under the applied tilted magnetic field as a function of the angle and the well width. It has been found in Ref. [28] that the direction of the magnetic field causes important changes in the binding energy which is approximately about $13 \mathrm{meV}$ when the angle changes from $15^{\circ}$ to $45^{\circ}$ for the width equals to $10 \mathrm{~nm}$. In addition, Zhang et al. have investigated the magnetic field effect on the binding energy of exciton in GaAs/(Ga,Al)As square QWW with infinite and finite potential barriers [29]. They have shown that the ground state exciton binding energy increases with the increasing of the magnetic field and the effect of a magnetic field is much stronger in wide quantum wire. In the previous work, we have shown that the ground-state probability density in $\mathrm{x}-\mathrm{y}$ plane maximizes at a point and starts to spread toward the boundaries as a function of the magnetic field [30]. We have also shown that the ground-state binding energy increases with the increase of the magnetic field and it maximizes for the effective radius around the effective Bohr radius.

In this work, based on the finite difference method adopted in our previous works $[30,31]$ and quasi-one dimensional effective potential model, the calculation of the lowest excited-state shallow donor binding energy in InGaN/GaN QWWs with lateral parabolic potential subjected to magnetic field parallel to wire-axis is investigated.

\section{THEORETICAL FRAMEWORK}

Let us assume that the hydrogenic shallow donor impurity is located at $\left(x_{i}, y_{i}\right)$ in the $(x, y)$-plane which constitutes the lateral surface of parabolic quantum-well wire (PQWW) made out of wurtzite (WZ) $\operatorname{In}_{\nu} \mathrm{Ga}_{1-\nu} \mathrm{N}$ and embedded in GaN barriers. Therefore, the electron is free to move along the $\mathrm{z}$-axis longitudinal direction and it is confined by the parabolic lateral confinement in $(\mathrm{x}, \mathrm{y})$ plane. In the presence of the hydrogenic shallowdonor impurity and a magnetic field applied parallel to the z-axis, the effective-mass Hamiltonian of an electron in PQWW can be expressed as follows

$$
H=-\frac{1}{2 m^{*}}\left[\vec{P}+\frac{e}{c} \vec{A}\right]^{2}+\frac{1}{2} m^{*} \omega_{0}^{2}\left(x^{2}+y^{2}\right)-\frac{e^{2}}{\varepsilon^{*} r_{i}}
$$

$e$ and $m^{*}\left(m^{*}=\nu \cdot m_{\mathrm{InN}}^{*}+(1-\nu) \cdot m_{\mathrm{GaN}}^{*}\right)$ are the electron charge and the electron effective-mass respectively, $\omega_{0}$ is the harmonic oscillator frequency, $\vec{P}$ is the momentum while $\varepsilon^{*}\left(\varepsilon^{*}=\nu \cdot \varepsilon_{\mathrm{InN}}^{*}+(1-\nu) \cdot \varepsilon_{\mathrm{GaN}}^{*}\right)$ is the dielectric constant of $(\mathrm{In}, \mathrm{Ga}) \mathrm{N}$ QWWs. $r_{i}$ is the electron-impurity distance in which $x_{i}$ and $y_{i}$ are the coordinates of the impurity in the PQWW given as::

$$
r_{i}=\sqrt{\left(x-x_{i}\right)^{2}+\left(y-y_{i}\right)^{2}+z^{2}}
$$


We note that $\vec{A}$ represents the vector potential of the magnetic field which is expressed as follows:

$$
\vec{A}=\frac{1}{2} \vec{B} \wedge \vec{r} \text { and } r=\sqrt{x^{2}+y^{2}+z^{2}}
$$

By using the effective Bohr radius (EBR) $a^{*}=\varepsilon^{*} \hbar^{2} / m^{*} e^{2}$ as the length unit and the effective Rydberg energy (ERE) $R^{*}=e^{2} / 2 \varepsilon^{*} a^{*}$ as the unit of the energy, the Hamiltonian (1) becomes:

$$
\begin{gathered}
H=-\nabla^{2}+\frac{\rho^{2}}{\rho_{e}^{4}}+\frac{\hbar \omega_{m}}{2 R^{*}} L_{z}-\frac{2}{r_{i}} \\
\rho_{e}=\frac{r_{0}}{\left[1+\frac{\omega_{m}^{2}}{4 \omega_{0}^{2}}\right]^{1 / 4}}
\end{gathered}
$$

$r_{0}=\sqrt{\frac{\hbar}{m^{*} \omega_{0}}}$ is the oscillator length and $\omega_{m}=\frac{e B}{m^{*} c}$ is defined as the cyclotron frequency.

In Eq. (4), $\rho$ is the electron distance from the origin of the wire along the z-axis. It is given as:

$$
\rho=\sqrt{x^{2}+y^{2}}
$$

For a fixed finite parabolic potential $\omega_{0}$, the value of $\rho_{e}$ can be modulated by the indium fraction in the QWW and the changeable magnetic field intensity. Note that the operator $L_{\mathrm{z}}$ is the angular momentum component of an electron which equals zero for the ground-state. We also mention that the lateral confinement effect scales as $\rho_{e}^{-4}$. It is also indicated that the confinement potential depends strongly on the value of $\rho_{e}$, i.e., the smaller the $\rho_{e}$, the stronger is the lateral confinement. Therefore, $\rho_{e}$ is defined as the effective radius (ER) of the wire cross section describing the lateral confinement of an electron in the PQWW.

By solving the Schrodinger equation in cylindrical coordinates in the absence of the donor impurity:

$$
H_{0} \psi_{0}^{\mathrm{ex}}(\vec{r})=E_{0}^{\mathrm{ex}} \psi_{0}^{\mathrm{ex}}(\vec{r})
$$

The exact solution of Eq. (7) is given as:

$$
\begin{gathered}
\psi_{0}^{\operatorname{ex}}(\rho, \theta)=\frac{1}{\sqrt{\pi} \rho_{e}^{2}} \rho \exp \left(-\rho^{2} / 2 \rho_{e}^{2}\right) \exp (i \theta) \\
E_{0}^{\mathrm{ex}}=\frac{4}{\rho_{e}^{2}}+\frac{\hbar \omega_{\mathrm{m}}}{2 R^{*}} L_{\mathrm{z}}
\end{gathered}
$$

In the presence of the impurity, there is no exact solution for the combination of Coulomb interaction and parabolic confinement potential. However, in the present situation we have adopted the same method used in Ref [32] in which the authors have proposed an analytical 1D formula for the effective interaction potential between confined carriers. Therefore, it is permissible to replace the Colombian potential with the effective potential energy $V_{\mathrm{eff}}^{\mathrm{ex}}(z)[32]$. Within this approximation, the Hamiltonian can be separated in the cylindrical coordinates and given:

$$
H=H_{\perp}^{\mathrm{ex}}+H_{/ /}^{\mathrm{ex}}
$$


$H_{\perp}^{\mathrm{ex}}$ is the perpendicular Hamiltonian which is the same as given in the absence of the impurity $\left(H_{\perp}^{\mathrm{ex}}=H_{0}\right)$ and $H_{/ /}^{\mathrm{ex}}$ is the parallel Hamiltonian given as:

$$
H_{/ /}^{\mathrm{ex}}=-\frac{d^{2}}{d z^{2}}-V_{\mathrm{eff}}^{\mathrm{ex}}(z)
$$

And the effective potential energy $V_{\mathrm{eff}}^{\mathrm{ex}}(z)$ is defined as:

$$
V_{\mathrm{eff}}^{\mathrm{ex}}(z)=\int_{-\infty}^{+\infty} \frac{2}{\varepsilon^{*} r_{i}}\left|\psi_{0}^{\mathrm{ex}}(x, y)\right|^{2} d x d y
$$

This integral (12) is calculated as done in Ref. [32] by replacing $\left(\left|\psi_{0}^{\mathrm{ex}}(x, y)\right|^{2}\right)$ and the Coulombian potential by their Fourier transforms, and integrating over the transverse coordinates and transverse momenta. Then, the effective interaction potential becomes expressed as the integral:

$$
V_{\mathrm{eff}}^{\mathrm{ex}}(z)=2 \int_{0}^{+\infty} J_{0}\left(u \sqrt{x_{i}^{2}+y_{i}^{2}}\right) \cdot\left(1-\frac{u^{2} \rho_{e}^{2}}{4}\right) \cdot \exp \left(-\frac{\rho_{e}^{2} u^{2}}{4}-u|z|\right) d u
$$

$J_{0}$ is the zeroth-order Bessel function and $\left|\psi_{0}^{\mathrm{ex}}(x, y)\right|^{2}$ is the lowest excited-state probability density of electron without the presence of the impurity in the QWWs.

The eigenvalues of the Hamiltonian given in Eq. (11) can be obtained using the finite-difference method on a one-dimensional mesh. The lowest excited-state energy with the presence of the impurity is given:

$$
E_{\mathrm{I}}^{\mathrm{ex}}=E_{0}^{\mathrm{ex}}+E^{\mathrm{ex}} / /
$$

And then, the lowest excited-state binding energy is obtained as follows:

$$
E_{b}^{\mathrm{ex}}=E_{0}^{\mathrm{ex}}-E_{\mathrm{I}}^{\mathrm{ex}}=-E_{/ /}^{\mathrm{ex}}
$$

To simplify, our results are given in the effective units $\left(a^{*}=2.80 \mathrm{~nm}\right.$ and $R^{*}=$ $26.65 \mathrm{meV}$ for $\operatorname{In}_{0.2} \mathrm{Ga}_{0.8} \mathrm{~N}$ ternary alloy).

\section{RESULTS AND DISCUSSION}

To get a clear picture of the lowest excited-state shallow donor binding energy dependence as a function of the magnetic field, the indium concentration and the parabolic potential, we have started to study the electron lowest excited-state probability density without the presence of the impurity.

Fig. 1 shows the magnetic field (effective radius) effect on the lowest excited-state probability density $\left|\psi_{0}^{\text {ex }}(x, y)\right|^{2}$ in $\mathrm{x}-\mathrm{y}$ plane without the presence of the impurity. It is shown that $\left|\psi_{0}^{\mathrm{ex}}(x, y)\right|^{2}$ is the smallest at a point on the center axis of the QWW. As the distance from the center axis increases, the probability density increases. When the distance reaches $\rho_{e},\left|\psi_{0}^{\text {ex }}(x, y)\right|^{2}$ is the largest and then it decreases as a function of the distance, i.e., $\left|\psi_{0}^{\mathrm{ex}}(x, y)\right|^{2}$ is maximum on the circularity $\left(C^{\mathrm{m}}\right)$ of radius $\rho_{e}$. The smallest $\rho_{e}$ is, the electrons are forced to be near the center axis but not on it. From the results obtained in our previous work, it appears that $\left|\psi_{0}^{\mathrm{ex}}(x, y)\right|^{2}$ has a complete behavior of the ground-state probability density [30]. 

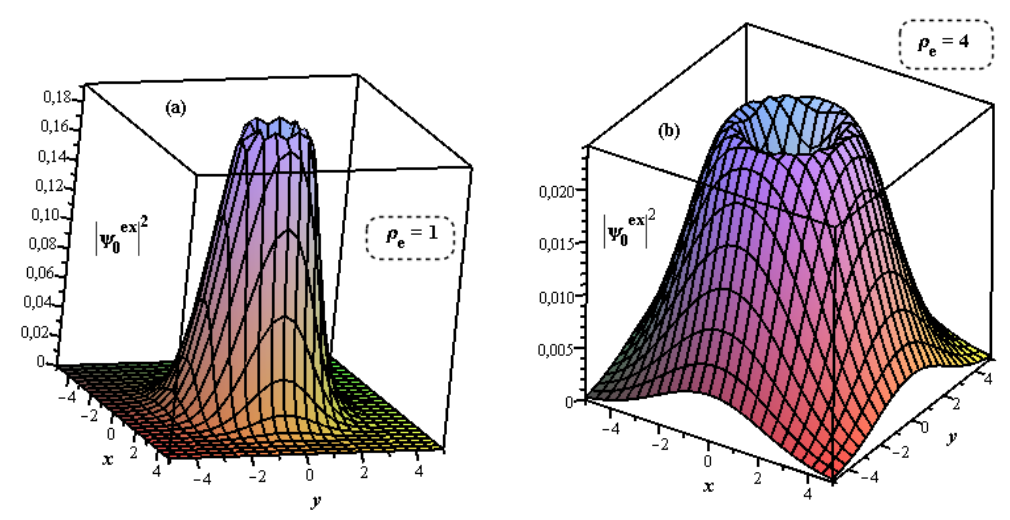

Fig. 1. (a and b): The electron lowest excited-state probability density in $x-y$ plane for two confinement regimes: (a) for $\rho_{e}=1$ and (b) for $\rho_{e}=4$ in $\mathrm{In}_{0.2} \mathrm{Ga}_{0.8} \mathrm{~N} / \mathrm{GaNPQWW}$.

In Fig. 2, the magnetic field effect on the lowest excited-state binding energy of shallow donor impurity located along the x-axis as a function of the impurity position is presented. The same results are obtained for the impurity located along the y-axis (not shown here). A typical symmetric behavior is exhibited around $x_{i}=0$. An obvious increase of the binding energy is shown especially for the impurity located around the center axis. A critical value of the effective radius is obtained $\rho_{e}^{c}=0.5$. It has been found that for $\rho_{e} \succ \rho_{e}^{c}$ the binding energy presents two maxima while for $\rho_{e} \prec \rho_{e}^{c}, E_{b}^{\mathbf{e x}}$ has only one. When the impurity is located on $C^{m}$, the probability density is the largest, i.e. the distance between the impurity and the electron is the shortest. Thus, when the impurity is located at $x_{i}= \pm \rho_{e}$ the binding energy is the largest. When the impurity is located within $\left(\rho \prec \rho_{e}\right)$ the $C^{m}, E_{b}^{\text {ex }}$ increases as a function of the position $\left|x_{i}\right|$. For the impurity located away from the $C^{m}$, the $E_{b}^{\mathbf{e x}}$ starts to decrease. Notice that the presence of the applied magnetic field parallel to the QWW axis leads to an increase of the binding energy. Such increase is also related to the indium concentration and the parabolic potential. The largest the indium concentration is, the larger the decrease is obvious. In addition, the largest the parabolic potential is, the larger the increase is.

It is well known that the increase of the magnetic field enhances the localization of the wave-function and then it acts as the reduction of the QWW geometrical dimension (effective radius). The results we presented above, corresponding to the effect of external magnetic field on the lowest excited binding energy, are in good agreement with those reported by several authors. We note that all of these works concern the ground-state and exciton state in different semiconductor materials (especially for GaAs, (Al,Ga)As, ZnS, InAs...), different shape and different numerical methods adopted. Incidentally, Hong and coworkers have investigated the magnetic field effect on the binding energy in GaAs QD [33] and GaAs double QD [34]. The same behavior as described above is reported especially for the QD but in self-assembled double quantum-dot (DQD) it is found that 


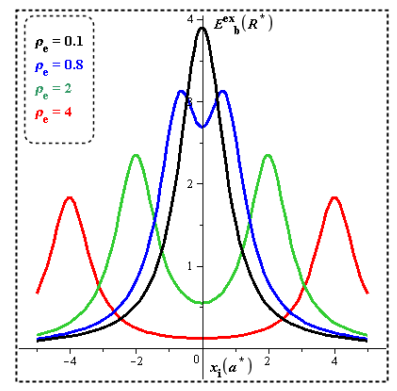

Fig. 2. The magnetic field effect on the lowest excited-state binding energy of shallow donor impurity located along the x-axis as a function of the impurity position in $\mathrm{In}_{0.2} \mathrm{Ga}_{0.8} \mathrm{~N} / \mathrm{GaNPQWW}$.

the binding energy has a complex behavior due to the coupling between the two dots, i.e., the magnetic field has different effects for different dot-size and for different lateral confinement. Recently, the same results concerning the magnetic field investigation on the binding energy of the ground state and the excited states have obtained by Abramov et al. [35] which has used the Green's function to obtain the solution of the Schrödinger equation in a square QW. For other shape like an InAs Pöschl-Teller quantum ring, the same behavior is also reported by Barseghyan et al. [36] for the ground state binding energy.

\section{CONCLUSION}

Within the framework of the effective-mass approximation and the quasi-onedimensional effective potential model, the magnetic field effect is investigated using the finite-difference method on the electron lowest excited-state probability density and the lowest excited-state shallow donor binding energy in parabolic-transversal section WZ $\mathrm{In}_{0.2} \mathrm{Ga}_{0.8} \mathrm{~N} / \mathrm{GaNPQWW}$. In this model, an effective radius is introduced which can be modulated by the Indium concentration, the parabolic potential and the magnetic field. The numerical results show that:

The electron lowest excited-state probability density is the largest on a circularity whose radius is the effective radius.

The lowest excited-state shallow donor binding energy is the largest when the impurity is located on this circularity while it starts to decrease as the impurity is away from the circularity.

\section{REFERENCES}

[1] N. Esuanu, E. C. Niculescu, L. M. Bureleanu, Physica E 41 (2009) 1386

[2] N. Esuanu and E. C. Niculescu, U. P. B. Sci. Bull. 72 (2010) 21-26

[3] P. Baser, I. Altuntas, S. Elagoz, Fen Bilimleri Dergisi 23 (2011) 171-180

[4] P. Baser, S. Elagoz, N. Baraz, Physica E 44 (2011) 356-360

[5] P. Baser, S. Elagoz, D. Kartal, H.D. Karki, Superlatt. Microstruct. 49 (2011) 497-503

[6] P. G. Moses, C. G. Van De Wall, Appl. Phys. Lett. 96 (2010) 021908-021910 
[7] E. Tangarife, S.Y. Lopez, M. de Dios-Leyva, L. E. Oliveira, C. A. Duque, Microelect. J. 39 (2008) 431-434

[8] I. D. Mikhailov, F. J. Betancur, R. A. Escorcia, J. Sierra-Ortega, Phys. Rev. B 67 (2003) 115317

[9] C.Y. Hsieh, J. Appl. Phys. 92 (2002) 484

[10] Y. Sidor, B. Partoens, F.M. Peeters, Phys. Rev. B 71 (2005) 165323

[11] P. Villamil, C. Cabra, N. Porras-Montenegro, Microelect. J. 39 (2008) 466

[12] S.T. Perez-Merchancano, R. Franco, J. Silva-Valencia, Microelect. J. 39 (2008) 383

[13] S. Aktas, F. K. Boz, S.S. Dalgic, Physica E 28 (2005) 96

[14] F. K. Boz, S. Aktas, Superlatt. Microstruct. 37 (2005) 63

[15] C. X. Xia, F.C. Jiang, S.Y. Wei, X. Zhao, Microelect. J. 38 (2007) 663

[16] C. I. Mendoza, G. J. Vazquez, M. del Castillo-Mussot, H. N. Spector, Phys. Rev. B 71 (2005) 075330

[17] S. Baskoutas, Phys. Lett. A 341 (2005) 303-307

[18] I. F. I. Mikhail, S. B. A. E. Sayed, Physica E 42 (2010) 2307-2313

[19] A. K. Manaselyan, A. A. Kirakosyan, Physica E 28 (2005) 462-470

[20] S. Baskoutas, A. F. Terzis, Eur. Phys. J. B 69 (2009) 237-244

[21] S. Baskoutas, and A. F. Terzis, J. Comput. Theor. Nanosci. 7 (2010) 492-497(4)

[22] S. Baskoutas, A. F. Terzis, E. Voutsinas, J. Comput. Theor. Nanosci. 1 (2004) 315-319

[23] Z. Zaiping, S. G. Christos, S. Baskoutas, J. Phys. D 45 (2012) 235102

[24] A. Zounoubi, K. E. Messouadi, A. Jorio, Superlatt. Microstruct. 30 (2001) 189

[25] K. Ryczko, G, Sek, J. Misiewicz, Superlatt. Microstruct. 32 (2002) 73

[26] X. T. An, J. J. Liu, J. Appl. Phys. 99 (2006) 123713

[27] C. E. Niculescu, L. Burileanu, U.P.B. Sci. Bull. 70 (2008) 55-62

[28] E. Kasapoglu, H. Sari, I. Sokmen, Sol. Stat. Commun. 125 (2003) 429-434

[29] Y. T. Zhang, B. Di, Z. Xie, Y. C. Li, Chin. Phys. Lett. 21 (2004) 166

[30] H. El Ghazi, Anouar Jorio, Izeddine Zorkani, Physica B (Revised form) 2013

[31] H. El Ghazi, I. Zorkani, A. Jorio, Physica B 412 (2013) 87-90

[32] S. Bednarek, B. Szafran, T. Chwiej, J. Adamowski, Phys. Rev. B 68 (2003) 045328

[33] Z. Hong, Z. Li-Xue, W. Xue, Z. Chun-Yuan, L. Jian-Jun, Chin. Phys. B 3 (2011) 037301

[34] Z. Hong, Z. Li-Xue, W. Xue, Z. Chun-Yuan, L. Jian-Jun, Chin. Phys. B 12 (2011) 127301

[35] A. Abramov, World. J. Cond. Matt. Phys. 2 (2012) 188-191

[36] M. G. Barseghyan, M. E. Mora-Ramos, C. A. Duque, Eur. Phys. J. B 84 (2011) 265-271 